\title{
Sintering Effects in Na substituted Bi-(2212) Superconductor Prepared by a Polymer method
}

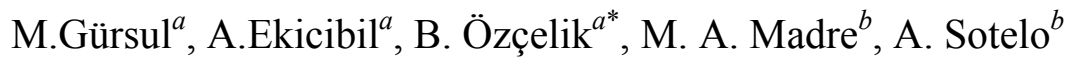 \\ ${ }^{a}$ Department of Physics, Faculty of Sciences and Letters, Çukurova University. 01330 Adana, \\ Turkey \\ ${ }^{b}$ ICMA (CSIC-Universidad de Zaragoza). María de Luna, 3. 50018 Zaragoza, Spain.
}

\begin{abstract}
In this study, $\mathrm{Na}$ substituted $\mathrm{Bi}_{2} \mathrm{Sr}_{2} \mathrm{Ca}_{0.9} \mathrm{Na}_{0.1} \mathrm{Cu}_{2} \mathrm{O}$ superconductor samples were prepared by a polymer solution method. Three different sintering temperatures $\left(850,860\right.$, and $\left.870^{\circ} \mathrm{C}\right)$ were used to study the effect of Na substitution. The samples have been characterized using X-ray diffraction, scanning electron microscopy (SEM), DC electrical resistivity and DC magnetic measurements. Magnetoresistivity measurements have shown a broadening of the superconducting transition under magnetic field which is explained on the basis of the Thermally Activated Flux Flow (TAFF) model. The calculated flux pinning energies of the samples varied from $0.17 \mathrm{eV}$ to $0.02 \mathrm{eV}$ by means of increasing magnetic field 0 to 9 Tesla. The upper critical magnetic field $H_{C 2}(0)$ and the coherence length $(\zeta(0))$ at $T=0 \mathrm{~K}$ were calculated using the resistivity data. $H_{C 2}(0)$ and $\xi(0)$ values have been calculated as 194,144 , 139 Tesla, and $15.5,15.2$ and $13 \AA$ for the 850,860 , and $870{ }^{\circ} \mathrm{C}$, respectively. TAFF model has shown $\mathrm{Bi}_{2} \mathrm{Sr}_{2} \mathrm{Ca}_{0.9} \mathrm{Na}_{0.1} \mathrm{Cu}_{2} \mathrm{O}_{8+\mathrm{y}}$ flux pinning energies are $0.015 \mathrm{eV}$ at $9 \mathrm{~T}$ in all cases, while they were $0.165,0153$, and $0.149 \mathrm{eV}$ at $0 \mathrm{~T}$ for samples sintered at 850,860 , and 870 ${ }^{\circ} \mathrm{C}$, respectively.
\end{abstract}

Keywords: Bi-based cuprates, XRD, SEM, Critical Current, M-H, Magnetoresitivity, TAFF, Pinning Force, Upper Critical Field

*Corresponding Author: Tel./fax:+90.322.3386060/2496/+90.322.3386070

e-mail: ozcelik@cu.edu.tr 


\section{Introduction}

The BSCCO family can be represented by the general $\mathrm{Bi}_{2} \mathrm{Sr}_{2} \mathrm{Ca}_{n-1} \mathrm{Cu}_{n} \mathrm{O}_{\mathrm{x}}$ formula, where $n=1,2$, or 3 , presenting $T_{c}$ 's of about 20,85 and $110 \mathrm{~K}$, respectively [1,2]. Here after, the abbreviations Bi-2201, Bi-2212 and Bi-2223 phases for $\mathrm{n}=1,2$, and 3, respectively will be used. Extensive experimental studies have been performed to understand the structural and physical properties of Bi-based superconductors [3-17]. It has also been well established that the superconducting properties are related to the hole concentration $[18,19]$ which is an essential parameter that controls many properties. Enormous efforts have been made to investigate the doping dependence of the superconducting properties and the mechanism of high temperature superconductors. Tarascon et al. [20] studied various substitutions of rare earths $(\mathrm{RE})$ in the $\mathrm{Bi}_{4} \mathrm{Sr}_{4} \mathrm{Ca}_{2-\mathrm{x}}(\mathrm{RE})_{\mathrm{x}} \mathrm{Cu}_{4} \mathrm{O}_{\mathrm{y}}$ system and reported that for $\mathrm{x}=1.5$ the compounds become semiconducting. At low doping $(x \leq 0.5) T_{c}$ was nearly unaffected. It is also concluded that the double $\mathrm{Bi}-\mathrm{O}$ layer acts as charge reservoir in the bismuth phases, and any modification affects the hole concentration and, as a consequence, resulting in a variation in $T_{c}$. Awana et al. [21] reported that the substitution of $\mathrm{Eu}, \mathrm{Dy}$, and $\mathrm{Tm}$ in the Bi-2212 system cause a transition from superconductor to insulator. Their studies gave an evidence of a transition from metallic behavior to a variable range hopping regime at high rare earth concentrations. Narsaiah et al. [22] showed that the Dy substitution in the Bi-2212 system was responsible for the magnetic properties in both the normal and superconducting states. The unique magnetic nature of rare-earths eases the study of interplay between the superconductivity and magnetism $[23,24]$. Earlier studies on the substitution of $\mathrm{Sm}$ for $\mathrm{Ca}$ showed that $\mathrm{T}_{\mathrm{c}}$ decreases as well as the other superconducting properties [25,26]. Variations in $T_{c}, J_{c}$ and lattice parameters were obtained by doping the system with other elements, at different proportions, and under various preparation conditions [27-31]. The substitution has also favoured the formation of Bi-2212 phase instead of Bi-2223 one. It was shown that $J_{c}$ decreased when the Bi-2212 phase proportion increases in Bi-2223 tapes [32]. Deng et al. [33] have observed that the residual $\mathrm{Bi}-2212$ phase is situated at grain boundaries of $\mathrm{Bi}-2223$ phase. But, one of the main concerns about the Bi-2212 superconductors is that the details of the current limiting mechanisms are not well understood.

In previous works, the effects of several substitutions for $\mathrm{Ca}$ or $\mathrm{Cu}$ in $\mathrm{Bi}-2212$ have been studied in detail $[3-7,16,17,34-36]$. On the other hand, in the best of our knowledge, no 
detailed studies about the Na-substitution effect on the Bi-2212 properties have been made. In previous works, the optimum Na-content to obtain the maximum amount of $\mathrm{Bi}-2212$ phase was determined [16]. In this work, the effect of the temperature on the Bi-(2212) phase formation and on the intergranular properties of polycrystalline of $\mathrm{Bi}_{2} \mathrm{Sr}_{2} \mathrm{Ca}_{0.9} \mathrm{Na}_{0.1} \mathrm{Cu}_{2} \mathrm{O}_{8+\mathrm{y}}$ sample prepared by polymer method will be determined. As a consequence, the aims of the present work are:

(i) determine the effect of a monovalent cation $(\mathrm{Na})$ partially substituting a divalent one $(\mathrm{Ca})$ in the crystal structure, and the changes in carrier concentration due to oxygen content modification.

(ii) exploring the structural, electrical and magnetoresistance behavior of these modified systems.

(iii) using a polymer solution method to prepare the samples due to the following reasons: (a) the particle size is smaller than for the classical solid state method, leading to higher reactivity of precursor powders, (b) $\mathrm{Na}_{2} \mathrm{CO}_{3}$, used for the solid state reaction, does not decompose at the treatment temperatures, only melts and it can be lost as a liquid phase during the thermal treatments, at least in a high proportion. As a consequence, it would be difficult to determine the substitution range, and (c) in the polymer solution method $\mathrm{Na}$ acetate is used (melting point $\sim 328^{\circ} \mathrm{C}$ ), but after coordination with polyethyleneimine (PEI) no sodium acetate can be found in the solution, as reported previously for the $\mathrm{Cu}$ acetate [37].

All these modification in Bi-2212 ceramics induced by the $10 \%$ of Na-substitution for Ca has been studied by powder X-ray analysis (XRD), scanning electron microscopy (SEM) and magnetoresistivity measurements.

\section{Experimental Procedure}

$\mathrm{Bi}_{2} \mathrm{Sr}_{2} \mathrm{Ca}_{0.9} \mathrm{Na}_{0.1} \mathrm{Cu}_{2} \mathrm{O}_{\text {y }}$ samples have been prepared, using a polymer matrix route from $\mathrm{Bi}\left(\mathrm{CH}_{3} \mathrm{COO}\right)_{3} \quad(\geq 99.99 \%, \quad$ Aldrich $), \quad \mathrm{Sr}\left(\mathrm{CH}_{3} \mathrm{COO}\right)_{2} \bullet 0.5 \mathrm{H}_{2} \mathrm{O} \quad$ (99\%, Panreac), $\mathrm{Ca}\left(\mathrm{CH}_{3} \mathrm{COO}\right)_{2} \cdot 2 \mathrm{H}_{2} \mathrm{O} \quad\left(98 \%\right.$, Alfa Aesar), $\mathrm{Cu}\left(\mathrm{CH}_{3} \mathrm{COO}\right)_{2} \cdot \mathrm{H}_{2} \mathrm{O} \quad(98 \%$, Panreac) and $\mathrm{Na}\left(\mathrm{CH}_{3} \mathrm{COO}\right)$ (99\%, Alfa Aesar) commercial powders as starting materials. They were weighed in stoichiometric amounts and dissolved in a mixture of glacial acetic acid $\left(\mathrm{CH}_{3} \mathrm{COOH}\right)$ (Panreac PA) and distilled water. The use of a mixture of glacial acetic acid and water is due to the fact that in one side $\mathrm{Sr}$ acetate is insoluble in concentrated acetic acid and, on the other side, $\mathrm{Bi}$ acetate is not soluble in water. Once a clear blue solution is obtained, 
polyethyleneimine (PEI) (Aldrich, $50 \mathrm{wt} \%$ water) was added. The mixture becomes dark blue immediately reflecting the formation of $\mathrm{Cu}-\mathrm{N}$ coordination bonds. The solution was then introduced into a rotary evaporator to reduce its volume (in $\sim 80 \%$ ), followed by heating on a hot plate at about $100{ }^{\circ} \mathrm{C}$ for total solvent evaporation, producing a thermoplastic dark blue paste. Further heating at around $350{ }^{\circ} \mathrm{C}$ produces a decomposition step (self combustion), as described schematically elsewhere [38], which produces the organic material decomposition. The resulting powder was then milled in an agate mortar and calcined twice at 750 and $800^{\circ} \mathrm{C}$ for $12 \mathrm{~h}$, with an intermediate manual milling, in order to decompose the alkaline-earth carbonates.

Finally, the prereacted homogeneous powders were pressed into $13 \mathrm{~mm}$ diameter pellets, and thermally treated in order to produce the Bi-2212 superconducting phase. This process was performed under air, and consisted in two steps: $60 \mathrm{~h}$ at three different sintering temperatures $850{ }^{\circ} \mathrm{C}, 860{ }^{\circ} \mathrm{C}$, and $870^{\circ} \mathrm{C}$, followed by $12 \mathrm{~h}$ at $800^{\circ} \mathrm{C}$ and, finally, quenched in air to room temperature.

In order to identify the present phases, powder X-ray diffraction patterns of the materials were recorded at room temperature using a Rigaku D/max-B powder diffractometer system working with $\mathrm{CuK} \alpha$ radiation and a constant scan rate between $2 \theta=3-60^{\circ}$. With these data, crystal lattice parameters were calculated with and error in the \pm 0.00001 range. SEM micrographs were taken using a LEO Evo-40 VPX scanning electron microscope fitted with an energy dispersive X-ray spectrometer (EDS). Magnetoresistivity measurements were carried out in a Quantum Design PPMS system. The samples sintering at $850{ }^{\circ} \mathrm{C}, 860{ }^{\circ} \mathrm{C}$, and $870^{\circ} \mathrm{C}$ will be hereafter named as $\mathrm{A}, \mathrm{B}$, and $\mathrm{C}$, respectively.

\section{Results and Discussions}

X-ray diffraction analyses were used to determine the phase and crystal structure of the samples. The XRD patterns of the A, B and C samples are shown in Fig. 1. At first sight, it is clear that the XRD patterns show the presence of 16 strong peaks, in all the samples. These XRD peaks are in good agreement with the standard ICPDS file for BSCCO. The sample A is mainly composed of Bi-2212 and Bi-2201 superconducting phases, with small amounts of $\mathrm{Bi}_{3} \mathrm{Sr}_{4} \mathrm{Cu}_{2} \mathrm{O}_{y}$, and $\mathrm{Na}_{2} \mathrm{O}$ non-superconducting ones. In the $\mathrm{B}$ and $\mathrm{C}$ samples, the $\mathrm{Bi}-2212$ phase is the major one. The X-ray diffraction patterns obtained for samples $\mathrm{B}$ and $\mathrm{C}$ 
indicate that with increasing sintering temperature the superconducting Bi-2212 phase amount is raised. As a result, increasing sintering temperature produces the decrease of Bi-2201 and non-superconducting phases. The calculated unit cell parameters for the Bi-2212 phase in the different samples are presented in Table-1. As it can be easily seen in Table-1, when increasing sintering temperature, a monotonic increase of $c$-parameter is produced, together with a modification of $a$ and $b$ parameters. These variations are due to the fact that the insertion of $\mathrm{Na}$ substituting $\mathrm{Ca}$ ions in the structure produces a decrease on the global positive charge in the structure. As a consequence, a modification on the oxygen content has to be produced to maintain the electrical neutrality in the structure, increasing c-parameter and, consequently, changing the $a-b$ ones and the cell volume.

Representative SEM micrographs and EDX spectra of all the samples are shown in Fig. 2a-c. In the figure, it can be clearly seen that the surface morphology of all the samples is roughly the same, independently of the sintering temperature. The particles shape and orientation, which appear as elongated platelets with no preferential orientation, is representative of all the samples and is typical for solid state sintered materials. On the other hand, B sample seems to show higher alignment than the observed in the other ones. EDX analyses (see Figs 2a-c) have shown that $\mathrm{Na}$ ions were successfully intergraded into the crystal structure. Moreover, the different phases identified by EDS are in agreement with the XRD data discussed previously.

The doping dependence of the transition temperature, $T_{c}$, of HTSC is fundamental interest for understanding the mechanism of the superconductivity. It is generally believed that one of the key parameters for controlling $T_{c}$ is the charge carrier concentration, $p$, in the $\mathrm{CuO}_{2}$ planes. Presland et al. [39] found the empirical relation;

$$
T_{c} / T_{c}^{\max }=1-82.6(p-0.16)^{2}
$$

between $T_{c}$ and Sr-hole doping in $\mathrm{La}_{1-\mathrm{x}} \mathrm{Sr}_{\mathrm{x}} \mathrm{CuO}_{4}$, where $T_{c}^{\text {max }}$ is the maximum attainable $T_{c}$, and $p$ is the hole concentration per $\mathrm{Cu}$ atom in the $\mathrm{CuO}_{2}$ planes. According to Eq.1, $T_{c}$ is maximized for hole concentrations $\approx 0.16$ and falls to zero at $\approx 0.05$ on the underdoped side and at $\approx 0.27$ on the overdoped side. The above relation has been found to be a remarkable model to determine the $T_{c}$ values versus doping concentration [40]. Therefore, the relation has often been used as a convenient representation of the relation between $T_{c}$ and the hole 
concentration for all the hole doped cuprates. The calculated $p$ values for the different materials are displayed in Table-1. These results show that the $p$-values are increased when sintering temperatures are raised. However, these values are close to the obtained in the undoped $\mathrm{Bi}-2223$ phase. These results indicate that the amount of $\mathrm{Na}$ dopant is far from the overdoping limit, in agreement with the results of Satyavathi et al. [41]. Therefore, the increase in the normal-state resistivity values ( $p$ at $T_{c}^{\text {onset }}$ ) and the decrease of $T_{c}$ can be attributed to the hole filling mechanism previously described by other groups [26,42].

The thermoelectric power, $S$, is highly sensitive to the charge transport mechanism, and hence information about the nature of the charge carriers, carrier concentration and band structure can be obtained. The studies of $S$ as a function of carrier concentration and temperature have been done in the Bi-based system [43]. It has been established that the high$T_{c}$ superconducting oxides are strongly correlated systems. The expression of $S$, at high temperatures was derived by different groups [44,45], and was modified by Cooper et al. [46]; according to these studies, the thermoelectric power of HTSC materials at high temperatures can be described by the formula;

$$
\left.\left.S=k_{B} / e[\ln (1-p) / 2 p)-\ln 2\right)\right]
$$

where $p, k_{B}$ and $e$ are the hole concentration, Boltzmann constant and electron charge, respectively. Using the $p$-values given in Table 1 , the $S$ values for the $\mathrm{A}, \mathrm{B}$, and $\mathrm{C}$ samples have been calculated as $-5.06 \times 10^{-4},-6.09 \times 10^{-4}$ and $-6.96 \times 10^{-4} \mu \mathrm{V} / \mathrm{K}$, respectively (see Table 1). As it can be seen, all the values are negative, clearly indicating a dominating electron conduction mechanism. Moreover, the absolute magnitude of $S$ increases when sintering temperature is raised, in agreement with the raise on the hole concentration which lead to a decrease on the electronic carriers.

It is well-known that the high- $T_{c}$ granular superconductors having a well defined superconducting transition temperature, generally display a two step resistive transition $\rho(T)$ and, correspondingly, $d \rho / d T$ displays a peak and a tail in the lower temperature side [47-49]. The peak temperature marks the superconducting transition within grains and the tail is related to the intergranular coupling. Fig. 3 shows the temperature dependence of resistivity for the A, B and C samples, with onset temperatures of about 92.1, 91.4 and, 91.2 K, and offset temperatures $78.9,78.2$, and $77.8 \mathrm{~K}$, respectively. The slight decrease in resistivity from room temperature to $90 \mathrm{~K}$ observed in all samples can be explained by the metallic 
character of the Bi-2212 phase at these temperatures. It can also be seen from these curves that the normal state resistivity regularly decreases from samples $\mathrm{A}$ to $\mathrm{C}$ and there is no tail regime clearly visible in $\rho(T)$. More information can be obtained by plotting the $d \rho / d T$ curves, as shown inset Fig. 3. The transition temperature, $T_{c}$, taken as the maximum of the $d \rho / d T$ curves is nearly the same for all samples (see Table 2). Moreover, all curves show a single peak, indicating a single superconducting transition. Furthermore, no hump in the low temperature side of the $d \rho / d T$ plot can be observed, revealing a clear indication of the strong link between grains.

In order to investigate in detail the effect of sintering temperature on the superconducting properties, magnetoresistivity measurements were performed in all the samples under different magnetic fields, and the results are plotted in Fig. 4. The transition temperatures deduced from these graphs are displayed in Table 2. Moreover, from these plots it can be observed that, as the field increases the tail of resistivity curves shifts to lower temperatures. The high temperature zone of resistivity remains practically unchanged, independently of the magnetic field. Furthermore, the broadening in the tail of the resistivity curves decreases from sample A to C. As it is well-known, the broadening and shifting of $T_{c}$ $(\rho=0)$ to lower temperature as a function of the magnetic field is proportional to the magnitude of the pinning force. In high- $T_{c}$ superconductors efficient pinning centers are produced by defects such as dislocations, twin planes, grain boundaries, radiation-induced columnar defects, etc [50-52]. The nature of pinning centers mainly depends on the grain boundaries, impurities and oxygen vacancies in $\mathrm{Cu}-\mathrm{O}$ layers. When the driving Lorentz force exceeds the pinning force, electric fields arises. Then, electrical resistance and hence energy dissipation occurs. At lower temperatures, a higher magnetic field is needed to depin the flux lines since lowering the temperature increases the pinning force [50]. This results in a shift of the zero resistivity temperature. $T_{c}^{\text {offset }}$ values significantly decrease with the applied magnetic field while $T_{c}^{\text {onset }}$ values do not significantly change, as generally observed for cuprates [53].

The study of thermally activated flux flow (TAFF) is manifested as a broadening of the superconducting transition. The resistivity in the TAFF region is caused by the thermally activated flux flow of the vortices. The experimental results in the tail part have been found to follow the Arrhenius relation as given by [54]

$$
\rho(H, T)=\rho_{0} \exp \left(-U(H) / k_{B} T\right)
$$


where $U$ is the flux pinning energy that depends on temperature and magnetic field. The $U$ value can be directly deduced from the slope of the plot of $\ln \left(\rho / \rho_{0}\right)$ versus $1 / T$. Fig. 5 shows the Arrhenius plots for all the samples. From these figures, it is clear that, as expected, the broadening for sample A is apparently reduced as compared to those of samples B and C. It is obvious that the resistive broadening caused by an applied magnetic field is reflected in a $T_{c}$ shift to lower temperatures. As mentioned above, this is a well-known behavior attributed to the flux flow resistivity. Since it is well established, lowering the temperature increases the pinning strength hence the pinning force. The other result which can be deduced from the semi-logarithmic Arrhenius plots of Fig. 5, is that indeed there is nearly an exponential dependence of $\rho$ on $1 / T$ for $T<<T_{c}^{\text {onset }}$, indicating the energy dissipation in the TAFF region due to the thermal activation of fluxes across the pinning barriers. Therefore, $U$ values are obtained from the slopes of the nearly straight line portions of the curves at low temperatures.

The calculated activation energy, $U$, from the linear fit in the tail part of the plots is presented in Fig.6 for all samples. As it can be seen in all cases, the activation energy decreases with the increase of applied magnetic field. The figure also shows that at the same field values, $U(H)$ for sample A are lower than those determined for samples B and C. Under magnetic fields below $\approx 2$ Tesla, the applied magnetic field can only penetrate the intergranular regions. On the other hand, a possible interpretation for the differences between the curves can be ascribed to the existence of different superconducting properties within the samples at the intragranular regions [55-57].

Fig. 7 shows the temperature dependence of the magnetization for all samples, zero field cooled, under an applied field of 50 Oe at temperatures below $100 \mathrm{~K}$. In all cases, the superconducting critical temperature $T_{c . o n}{ }^{m a g}$ defined as the onset of diamagnetism on the $M-T$ curves is about $85 \mathrm{~K}$, since the major phase is the Bi-2212 and most of the inductive transition takes place at lower temperatures. A very small anomaly is, however, observed in the M-T curves at about $85 \mathrm{~K}$, suggesting that the samples have not been completely transformed into the Bi-2212 phase at the higher sintering temperatures. This behavior is characteristic of all the bulk superconductors, mainly caused by their granular nature together with possible secondary phases, and thus the grain boundaries could then be considered as weak Josephson junction [58].

The magnetic hysteresis behavior of samples has also been studied. All the hysteresis loops were measured under applied fields between 9 Tesla at $10 \mathrm{~K}$. Typical examples of magnetic hysteresis $(M-H)$ loops for all the samples are shown in Fig. 8. The samples were 
warmed up above the superconducting transition temperature for each measurement in order to expel any trapped flux. Then the system was zero field cooled (ZFC) for each measurement. All the samples exhibit weak field dependence, particularly after 2T. The decrease of the hysteresis loops with increasing sintering temperature and the symmetrical behavior indicate the existence of flux pinning centers. Thus it might be argued that the magnetization behavior at low fields is dominated by the bulk pinning rather than surface and geometrical barriers.

The calculated critical current densities, $J_{c}$, of the samples as a function of the applied field are shown in Fig. 9. According to Bean's critical state model [59], $J_{c}$ is proportional to the width of a hysteresis loop and for slab geometry it can be expressed as

$$
J_{c}=20 \Delta M /[a(1-a / 3 b)]
$$

where $a$ and $b(a<b)$ are the sample dimensions perpendicular to the applied field, and $\Delta M$ is the so-called magnetization irreversibility, i.e. the width of hysteresis loop. In Fig. 9, it is clear that the general shape of $J_{c}$ is the typical for type-II superconductors. The critical current density is not an intrinsic parameter of a superconductor. Furthermore, in a system such as Bi2212 where surface effects are present, the critical currents in the material should be carefully considered. Nevertheless, the use of the Bean's critical state model can provide us with an indication of the strength of the pinning within the system. From the figure it is obvious that $J_{c}$ decreases with increasing the sintering temperature. In fact, the critical current density measured in these samples has strong field dependence and reaches a plateau of the order of $15 \mathrm{~A} / \mathrm{cm}^{2}$ around 2 Tesla. The strong field dependence of $J_{c}$ may be caused by the large thermally activated flux motion in the system. The result mentioned above indicates that the flux pinning is effective in the Bi-2212 system. The highest critical current density values at around zero field for samples A, B, and C are $48.10^{5}, 43.10^{5}$ and $42.10^{5} \mathrm{~A} / \mathrm{cm}^{2}$, respectively. It should be noted that in the critical state, $J_{c}$ is almost completely determined by imperfections in a superconducting material, and the large $J_{c}$ indicates the existence of strong flux pinning in the material. Therefore, the pinning in the studied system is stronger at low fields. This field dependence clearly demonstrates that the flux pinning decreases with the increasing field. The decrease in the magnitude of $J_{c}$ due to the raise in sintering temperature means that the contacts between the grains is poorer, limiting the super-current paths. However, the obtained results indicate that the increase in sintering temperature produces the 
formation of insulating layers in the grain boundaries. Probably because of this reason, the $J_{c}$ values of $\mathrm{B}$ and $\mathrm{C}$ samples are lower than that of the $\mathrm{A}$ one.

The $J_{c}$ data have been used to obtain the volume pinning force $F_{p}$ in the different compounds, using the formula:

$$
F_{p}=J_{c} \times B
$$

The plots of $F_{p}$ versus $H$ are shown in Fig. 10. The maximum values of $F_{p}$ appeared at $2 \mathrm{~T}$, indicating that the irreversibility line shifts to lower magnetic fields with increasing sintering temperature. This reinforces the previous discussions about the decrease of flux pinning strength when the sintering temperature is raised.

The calculated upper critical field, $H_{c 2}$ and the coherence length, $\xi$, versus the critical temperatures are plotted in Fig. 11. $H_{c 2}$ cannot be measured at low temperatures and, as a consequence, in order to determine the behavior of the upper critical field at temperatures close to zero, a theoretical model, defined by Werthamer-Helfand-Hohenberg (WHH) has been used, applying the formula [60]

$$
\mu_{0} H_{c 2}(0)=0.693 T_{c}\left(d \mu_{0} H_{c 2}(T) / d T\right)
$$

where the $\left(d H_{c 2}(T) / d T\right)$ are determined using the slopes at $T_{c}$ of curves in Fig 11. The calculated upper critical fields at zero K for H//c are 192.4, 143.8 and 136.8 Tesla for samples $\mathrm{A}, \mathrm{B}$, and $\mathrm{C}$, respectively. These are quite acceptable values for a good superconductor. Another important parameter in the superconductivity is the coherence length, $\xi$. In the framework of this study, the coherence length, $\xi$, has been calculated using the GinzburgLandau relation,

$$
\mu_{0} H_{c 2}(0)=\Phi_{0} / 2 \pi \xi^{2}(0)
$$

where $\Phi_{0}$ is the quanta of flux $\left(2.07 \times 10^{-15} \mathrm{~T} . \mathrm{m}^{2}\right)$ and $H_{c 2}(0)$ is the upper critical field at zero temperature. The calculated values of the coherence length at zero temperature has been found to be $13.080,15.136$ and $15.518 \mathrm{~nm}$ for samples A, B and C, respectively. 


\section{Conclusions}

In summary, a comparative study of physical and magnetic properties of $\mathrm{Bi}_{2} \mathrm{Sr}_{2} \mathrm{Ca}_{0.9} \mathrm{Na}_{0.1} \mathrm{Cu}_{2} \mathrm{O}_{\mathrm{y}}$ samples prepared by a polymer solution technique has been presented. X-ray diffraction, scanning electron microscopy, magnetoresistivity, dc-magnetization and magnetic hysteresis studies have been performed to find the optimum sintering temperature. XRD suggested that samples with nearly single Bi-2212 phase have been produced, independently of the sintering temperature. SEM micrographs confirmed that the surface morphology of the samples with different sintering temperatures are almost similar. All samples are predominantly composed by randomly oriented Bi-2212 plate-like grains, typical for solid state sintered materials. $R-T$ results indicate that all samples exhibit metallic behavior above $T_{c}^{\text {onset }}$, confirming that $\mathrm{Bi}-2212$ phase is the major one. The activation energies, irreversibility fields $\left(H_{\text {irr }}\right)$, upper critical fields $\left(H_{C 2}\right)$ and coherence lengths at $0 \mathrm{~K}(\zeta(0))$ were evaluated from the resistivity versus temperature $(\rho-T)$ curves, under DC magnetic fields up to $9 \mathrm{~T}$. The electrical resistivity broadening behavior under the applied magnetic field has been studied using the thermally activated flux creep model. From $M-H$ measurements, it has been found that the magnetization values and the volume of the closed hysteresis curves decrease with increasing sintering temperature due to the formation of the Na-substituted Bi-2212 phase. The decrease of the hysteresis loops with increasing sintering temperature and the symmetrical behavior indicate the existence of flux pinning centers. Thus, the magnetization behavior at low fields is dominated by the bulk pinning rather than surface and geometrical barriers.

Employing Bean's model, it has been found that the decrease on sintering temperature produce a raise of $J_{c}$ values. The highest $J_{c}$ values at zero field for samples A, B, and C have been determined to be $48.10^{5}, 43.10^{5}$ and $42.10^{5} \mathrm{~A} / \mathrm{cm}^{2}$, respectively.

In conclusion, with the increasing of the sintering temperature, the superconducting properties are deteriorated and the optimum sintering temperature to form the Bi-2212 phase has been determined to be $860{ }^{\circ} \mathrm{C}$ for these Na-substituted samples.

\section{Acknowledgements}

This work is supported by the Research Fund of Çukurova University, Adana, Turkey, under grant contracts no. FEF2013YL18. 


\section{References}

[1] C. Michel, M. Herviev, M.M. Borel, A. Grandin, F. Deslands, J. Provots, B. Raveav, Z. Phys. B86, 421 (1987)

[2] H. Maeda, Y. Tanaka, M. Fukutumi, T. Asano, Jpn. J. Appl. Phys. 27(2), 209 (1988)

[3] B. Özçelik, H.Gündoğmuş, D.Yazıcı, J Mater Sci: Mater Electron 25, 2456 (2014)

[4] B. Özçelik, C. Kaya, H. Gündoğmuş, A. Sotelo, M.A. Madre, J Low Temp Phys. $174,136(2014)$

[5] N. Türk, H. Gündogmus, M. Akyol, Z. D. Yakıncı, A. Ekicibil, B. Özçelik J Supercond Nov Magn27, 711 (2014)

[6] A. Özaslan, B. Özçelik, B. Özkurt, A. Sotelo,M.A. Madre, J Supercond Nov Magn 27, 53 (2014)

[7] H.Gündoğmuş, B. Özçelik, A. Sotelo, M. A. Madre J Mater Sci: Mater Electron 24, 2568 (2013)

[8] V. Lennikov, B. Özkurt, L. A. Angurel, A. Sotelo, B. Özçelik, G. F. de la Fuente, J Supercond.Nov.Magn. 26, 947 (2013)

[9] B. Özçelik, B. Özkurt, M.E. Yakıncı, A. Sotelo, M. A. Madre, J Supercond Nov Magn. $26,873(2013)$

[10] D. Yazıc1, B. Ozçelik, M.E. Yakınc1, J. Low Temp. Phys. 163, 370 (2011)

[11] A. Sotelo, M. Mora, M. A. Madre, J. C. Diez, L. A. Angurel, G. F. de la Fuente, J. Eur. Ceram. Soc. 25, 2947 (2005)

[12] M. Mora, A. Sotelo, H. Amaveda, M. A. Madre, J. C. Diez, L. A. Angurel, G. F. de la Fuente, Bol. Soc. Esp. Ceram. V. 44, 199 (2005)

[13] G. F. de la Fuente, A. Sotelo, Y. Huang, M. T. Ruiz, A. Badia, L. A. Angurel, F. Lera, R. Navarro, C. Rillo, R. Ibañez, D. Beltran, F. Sapiña, A. Beltran, Physica C185, 509 (1991)

[14] B. Özkurt, M. A. Madre, A. Sotelo, M.E. Yakıncı, B. Özçelik, J. Supercond. Nov. Magn. $25,799(2012)$

[15] S. Bal, M. Dogruer, G. Yıldırım, A. Varilci, C. Terzioglu, Y. Zalaoglu, J. Supercond. Nov. Magn. 25, 847 (2012)

[16] B. Özçelik, M.Gürsul, A. Sotelo, M. A. Madre, J Mater Sci: Mater Electron DOI:10.1007/s10854-014-2419-8

[17] B. Özçelik, M.Gürsul, A. Sotelo, M. A. Madre, J Mater Sci: Mater Electron DOI 10.1007/s10854-014-2190-x 
[18] J.B. Torrance, Y. Tokura, A.I. Nazzal, A.Bezinge, T.C. Huang and S.S.P. Parkin, Phys. Rev. Letters 61, 1127 (1988)

[19] Y.Takura, J.B. Torrance, T.C. Huang and A.I. Nazzal, Phys.Rev.B 38, 7156 (1988)

[20] J.M. Tarascon, P. Barboux, G.W. Hull, R. Ramesil, L.H. Greene, M. Giround, M.S.

Hegde, and W.R. McKinnon, Phys. Rev. B 39, 7156 (1989)

[21] V.P.S. Awana, S.K. Agarwal, R. Ray, S. Gupta, and A.V. Narlikar, Physica C 19143 (1992)

[22] E. Laxmi Narsaiah, U.V. SubbaRao, O. Pena, and C. Perrin, Solid State Commun., 83, $689(1992)$

[23] B. Jayaram, P.C. Lanchester, M.T. Weller, Physica C 160, 17 (1989)

[24] K. Nanda Kishare, S. Satyavathi, M. Muradlidhar, V. Hari Babu and O. Pena, Physica C 235 (1994)

[25] S. Satyavathi, M. Muradlidhar and K. Nanda Kishore, Appl. Supercond. 3, 187 (1995)

[26] K. Nanda Kishare, M. Muradlidhar, V. Hari Babu and O. Pena, Phsica C 204, 299 (1993)

[27] C. Terzioglu, M. Y1lmazlar, O. Ozturk, E. Yanmaz, Physica C 423, 119 (2005)

[28] C. Terzioglu, O. Ozturk, A. Kilic, A. Gencer, I. Belenli, Physica C 434, 153 (2006)

[29] A. Biju, R.P. Aloysius, U. Syamaprasad, Supercond. Sci. Technol. 18, 1454 (2005)

[30] A. Ekicibil, A. Coskun, B. Ozcelik, K. Kiymac, J.Low Temp. Phys. 140, 105 (2005)

[31] A. Ekicibil, A. Coskun, B. Ozcelik, K. Kiymac, Mod. Phys. Letters B, 19, 331 (2005)

[32] J. Jiang, X.Y. Cai, J.G. Chandler, S. Patnaike, A.A. Polyanskii, Y. Yuan, E.E.

Hellestrom, D.C. Larbalestier, IEEE Trans. Appl. Supercond. 13, 3018 (2003)

[33] H. Deng, P. Hua, W. Wang, C. Dong, H. Chen, F. Wu, H. Wang, Y. Zhov, G. Yuan, Physica C 339, 181 (2000)

[34] H. Gündogmus, B. Özçelik, B. Özkurt, A. Sotelo, M.A. Madre, J. Supercond. Nov. Magn, 26, 111(2013)

[35] C. Kaya, B. Özçelik, B. Özkurt, A. Sotelo, M.A. Madre, J Mater Sci:Mater Electron, 24, $1580(2013)$

[36] B.Özkurt, A.Ekicibil, M.A.Aksan, B.Özçelik, M E.Yakınc1, K.Kıymaç, Low Temp.Phys, 149, No:1/2 October, 105 (2007)

[37] A. Sotelo, G. F. de la Fuente, F. Lera, D. Beltran, F. Sapiña, R. Ibañez, A. Beltran, M. R. Bermejo, Chem. Mater. 5, 851 (1993)

[38] A. Sotelo, L. A. Angurel, M. T. Ruiz, A. Larrea, F. Lera, G. F. de la Fuente, Solid State Ionics 63, 883 (1993) 
[39] M.R.Presland, J.L. Tallon, R.G. Buckley, R.S. Liv, N.E. Floer, PhysicaC, 176, 95 (1991)

[40] J.B. Torrance, A. Bezinge, A.I. Nazzal, Phys. Rev. B 40, 8872 (1989)

[41] S. Satyavathi, K . Nanda Kishore, V. Hari Babu, O. Pena, Supercond. Sci. Technol., 9, 93, (1996)

[42] M. M. İbrahim, S. M. Khalil, A. M. Ahmed, J. Phys. Chem. Solids, 61, 1553 (2000)

[43] M. C. Sekhar and S.V. Suryanarayana, Physica C, 415, 209 (2004)

[44] G. Beni, Phys. Rev. B10, 2187 (1974)

[45] P.M.Chaikin, G.Beni, Phys. Rev.B 46, 647 (1976)

[46] J.R. Cooper, B. Alavi, L.W. Zhow, W.P. Boyermann, G. Gruner, Phys. Rev. B 35, 8794 (1987)

[47] H. Salamati, P. Kameli, Solid State Commun., 125, 407 (2003)

[48] A. R. Jurelo, I. A. Castiro, J.Roa-Rojas, L.M. Ferreira, L. Ghivelder, P. Pureur, P. Radriguresjr, Physcia C, 311, 133 (1999)

[49] P. Kameli, H. Salamati, M. Eslami, Solid State Commun., 137, 30 (2006)

[50] M. Tinkham, “Introduction to Superconductivity”, McGrow-Hill, Newyork, (1996)

[51] A. Junka, I. Barboy, G. Jung, S.S. Banerjee, Y. Myasoedov, V. Plausinaitiene, A.

Abrutis, X. Li, D. Wang, R. Sobolewski, Appl. Phys. Lett., 87, 192504 (2005)

[52] P. Kameli, H. Salamati, I. Abdolhosseini, D. Sohrabi, Physica C, 468137 (2008)

[53] C.S. Yadav, P. L. Paulose, New J. Phys., 11, 103046 (2009)

[54] J. J. Kim, H. Lee, J. Chung, H.J. Shin, H. J. Lee, J.K. Ku, Phys. Rev. B, 43, 2962 (1991)

[55] E. Govoea-Alcaide, J. Garcia-Fornaris, P. Mune, R.F. Jardim, Eur. Phys. J. B58, 373 (2007)

[56] D. Sharma, R. Kumar, V.P.S. Awana, Solid State Commun., 152, 941 (2012)

[57] M.Erdem, O. Ozturk, E. Yucel, S. P. Altintas, A. Varilci, C. Terzioglu, I. Belenli, Physica B, 406, 709 (2011)

[58] K. Gernot, F. Gunter, C. Wolf-Rüdiger, M.Hardo, P. Ryszard, "High Temperature Superconductor Bulk Material Fundamentals - Processing - Properties Control Application Aspects” Wiley-Vch Verlag GmbH-Co KgaA, (2006)

[59] C. P. Bean, Rev. Mod. Phys. 36, 31 (1994)

[60] N.R. Werhamer, E. Helfand, P.C. Hohenberg, Phys. Rev. 147, 295 (1966) 


\section{Figure captions}

Figure 1: XRD patterns for the A, B, and C samples.

Figure 2: SEM images and EDX spectra for the A, B, and C samples.

Figure 3: Electrical resistivity versus temperature for the $\mathrm{A}, \mathrm{B}$, and $\mathrm{C}$ samples.

Figure 4: Magnetic field effect on the resistivity as a function of temperature for the A, B, and $\mathrm{C}$ samples.

Figure 5: Arrhenius plot of the resistivity for the A, B, and C samples.

Figure 6: Flux pinning energy, U, versus applied magnetic field for all the samples

Figure 7: Magnetization as a function of temperature for all the samples at 50 Oe applied field

Figure 8: $\mathrm{M}-\mathrm{H}$ hysteresis loops for all the samples at $\mathrm{T}=10 \mathrm{~K}$

Figure 9: Calculated $J_{c}$ results for all the samples at $\mathrm{T}=10 \mathrm{~K}$

Figure 10: Variation of pinning force as a function of the applied magnetic fields

Figure 11: Coherence lengths and $\mathrm{H}_{\mathrm{c} 2}$ values variation with the sintering temperature 
Table 1. Structural and transport properties of the samples

\begin{tabular}{cccccc}
\hline $\begin{array}{c}\text { Samp. } \\
\text { code }\end{array}$ & $\mathbf{a}(=\mathbf{b})(\AA)$ & $\mathbf{c}(\AA)$ & Unit cell volume, $\left(\AA^{3}\right)$ & $\boldsymbol{p}$, Hole number & TEP, $\mathbf{~ ( 1 0 - 4} \mathbf{V} / \mathbf{K})$ \\
\hline A & 5.3891 & 30.3901 & 882.6014 & 0.1741 & -5.06 \\
\hline B & 5.3573 & 30.7013 & 881.1476 & 0.1783 & -6.09 \\
\hline C & 5.4014 & 30.7861 & 898.1882 & 0.1809 & -6.96 \\
\hline
\end{tabular}


Table 2. $\mathrm{T}_{\text {c.onset }}$ and $\mathrm{T}_{\text {c.offset }}$ values of the samples at various applied magnetic field.

\begin{tabular}{|c|c|c|c|c|c|c|}
\hline Sample & 0 Tesla & 1 Tesla & 3 Tesla & 5 Tesla & 7 Tesla & 9 Tesla \\
\hline $\begin{array}{ll}\text { A } & \mathrm{T}_{\text {c.onset }}\end{array}$ & $92,06 \mathrm{~K}$ & $91,98 \mathrm{~K}$ & $91,56 \mathrm{~K}$ & $91,01 \mathrm{~K}$ & $90,32 \mathrm{~K}$ & $89,80 \mathrm{~K}$ \\
\hline $\mathrm{T}_{\text {c.offset }}$ & $78,90 \mathrm{~K}$ & $64,37 \mathrm{~K}$ & $55,31 \mathrm{~K}$ & $49,98 \mathrm{~K}$ & $46,88 \mathrm{~K}$ & $43,93 \mathrm{~K}$ \\
\hline B $\mathrm{T}_{\text {c.onset }}$ & $91,44 \mathrm{~K}$ & $90,89 \mathrm{~K}$ & $90,26 \mathrm{~K}$ & $89,36 \mathrm{~K}$ & $88,62 \mathrm{~K}$ & $87,85 \mathrm{~K}$ \\
\hline $\mathrm{T}_{\text {c.offset }}$ & $78,24 \mathrm{~K}$ & $62,80 \mathrm{~K}$ & $53,03 \mathrm{~K}$ & $48,50 \mathrm{~K}$ & $44,68 \mathrm{~K}$ & $42,40 \mathrm{~K}$ \\
\hline C $\mathrm{T}_{\text {c.onset }}$ & $91,17 \mathrm{~K}$ & $90,82 \mathrm{~K}$ & $90,03 \mathrm{~K}$ & $89,32 \mathrm{~K}$ & $88,54 \mathrm{~K}$ & $87,77 \mathrm{~K}$ \\
\hline$T_{\text {c.offset }}$ & $77,81 \mathrm{~K}$ & $62,00 \mathrm{~K}$ & $52,96 \mathrm{~K}$ & $47,62 \mathrm{~K}$ & $44,59 \mathrm{~K}$ & $41,54 \mathrm{~K}$ \\
\hline
\end{tabular}


Figure 1

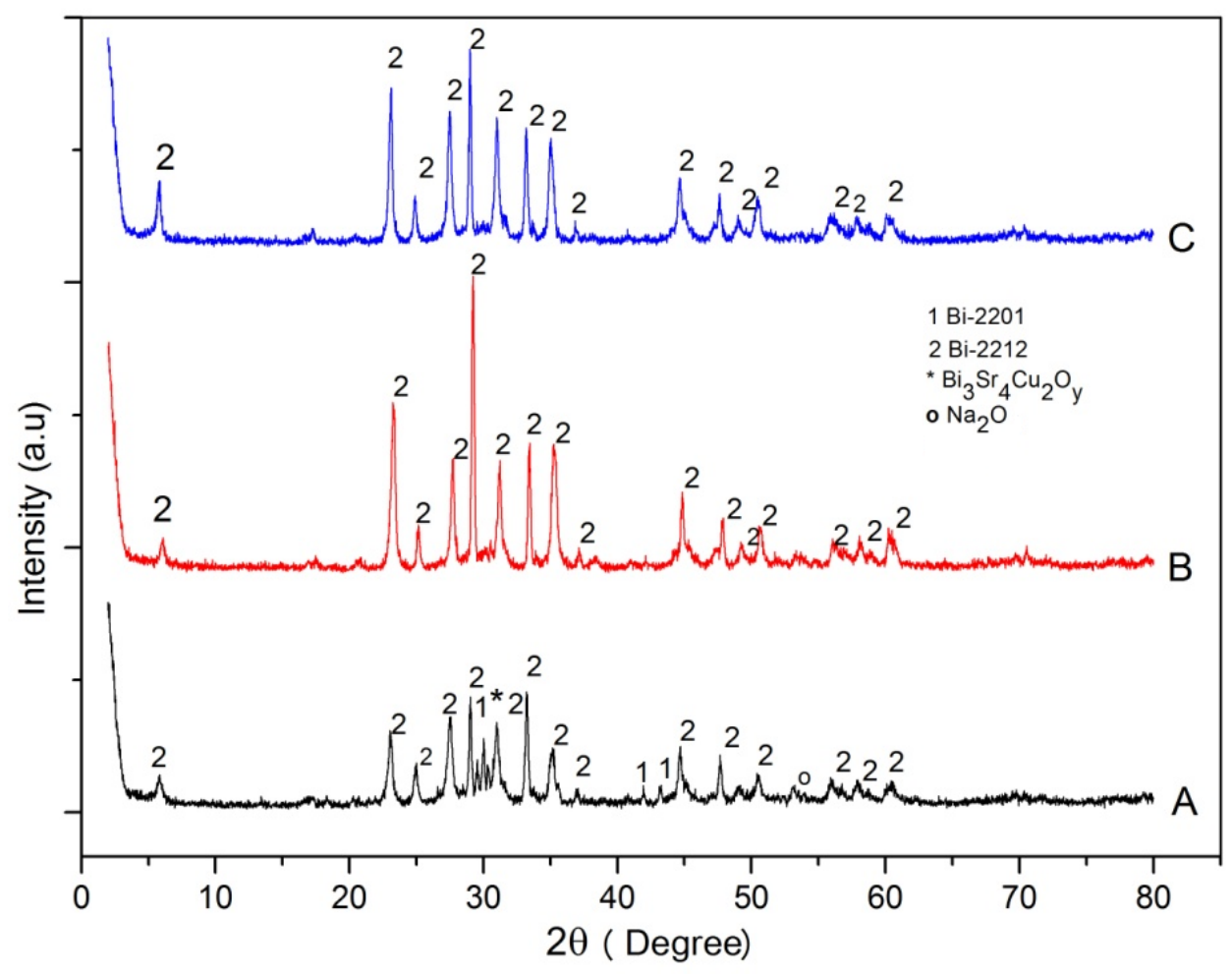


Figure 2
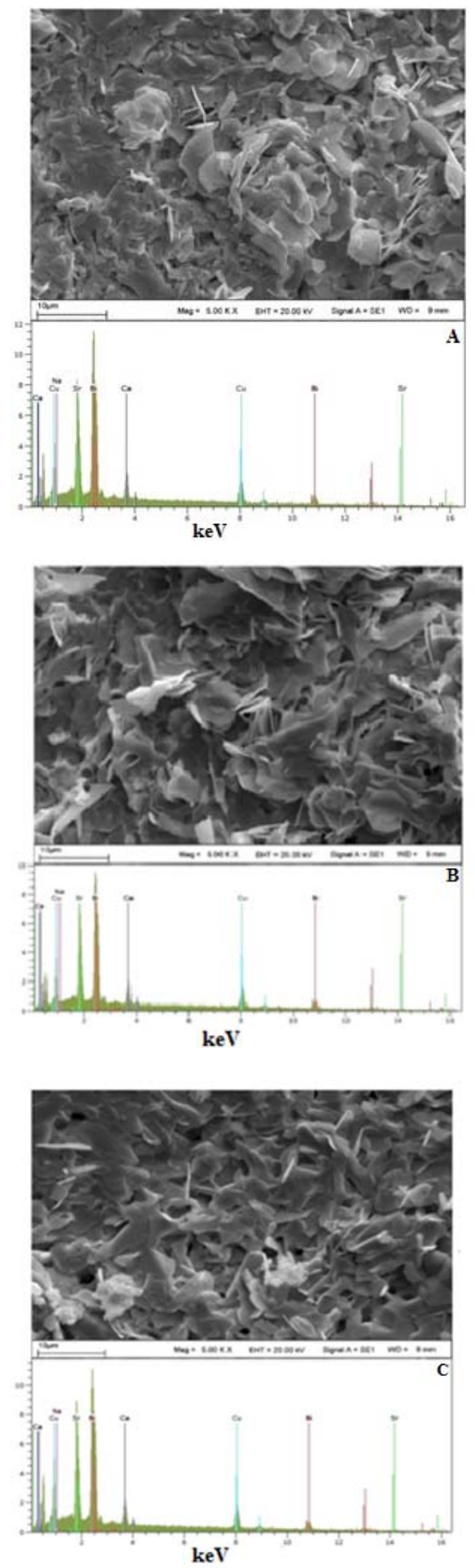
Figure 3

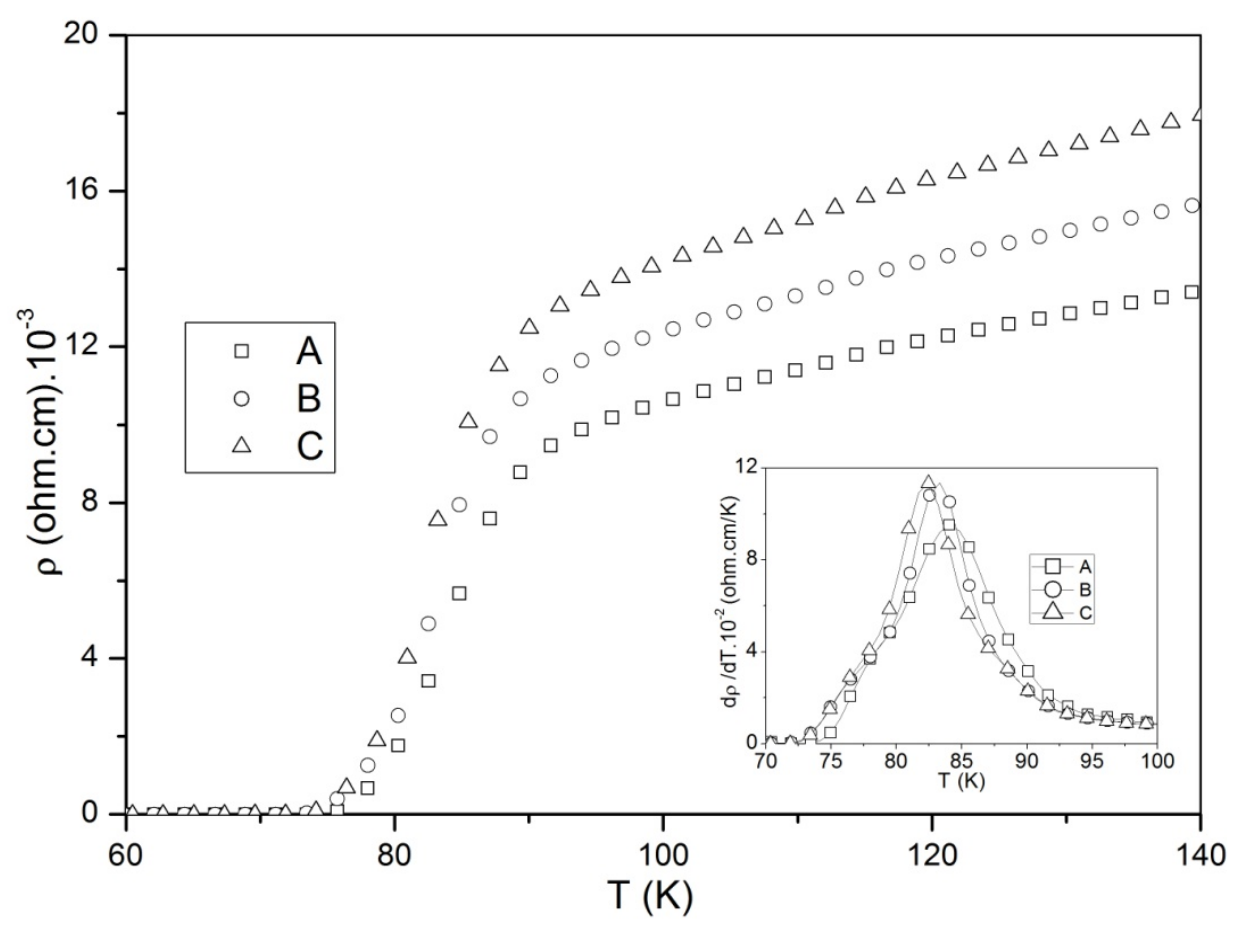


Figure 4
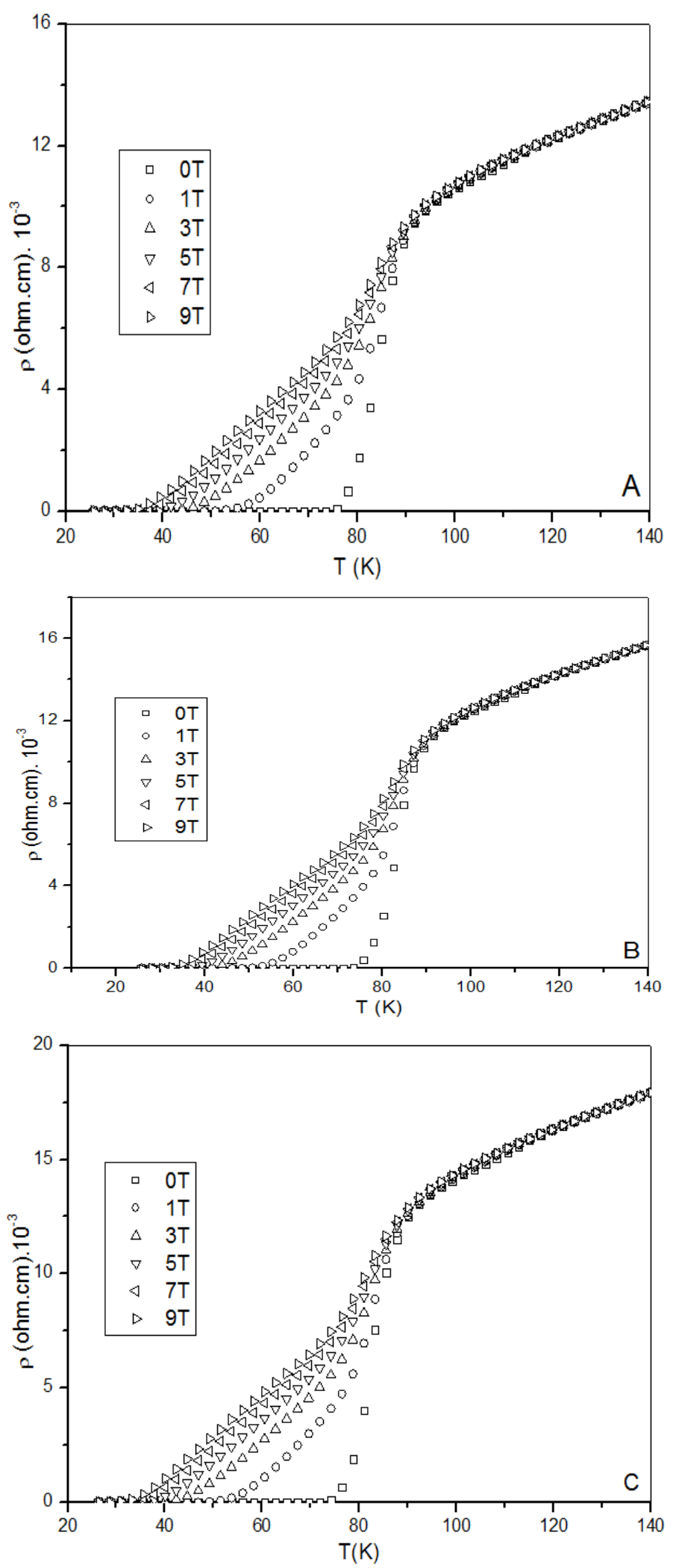
Figure 5
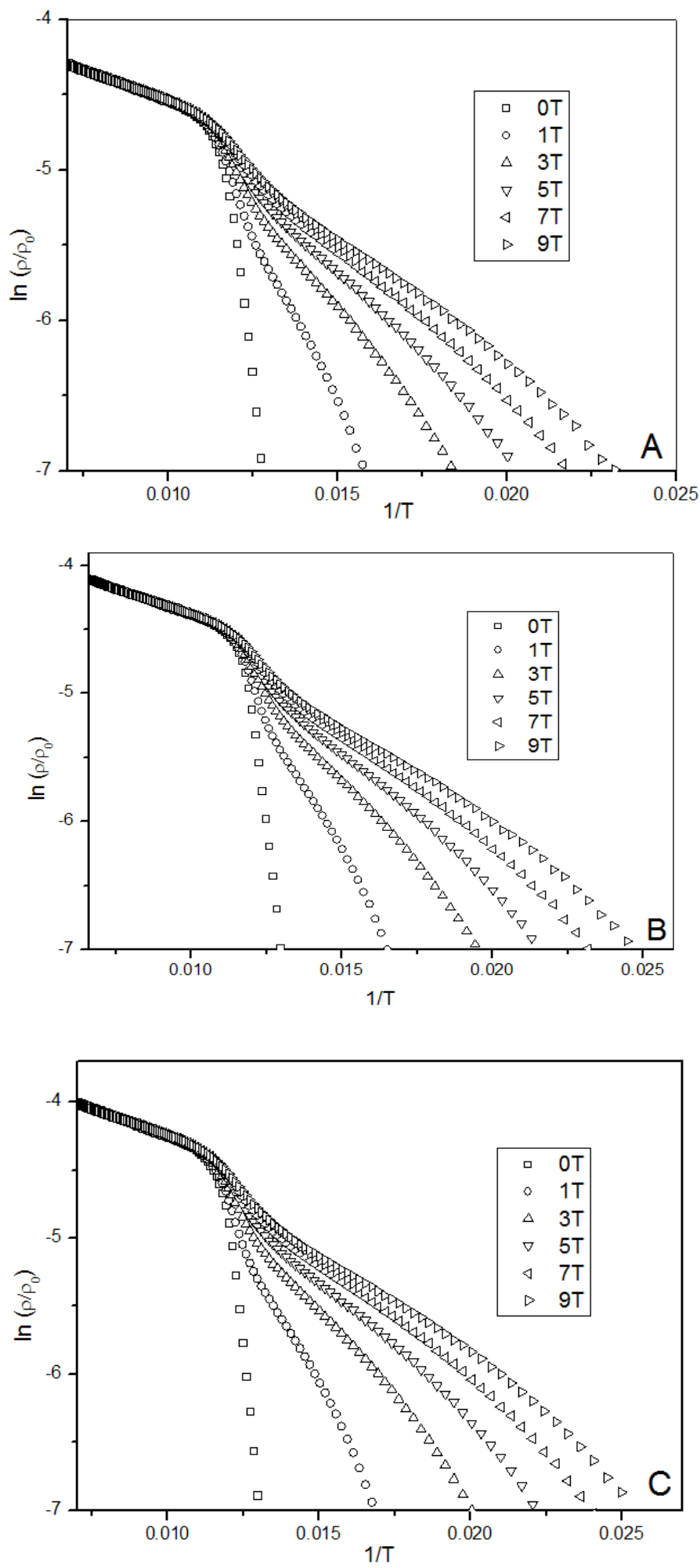
Figure 6

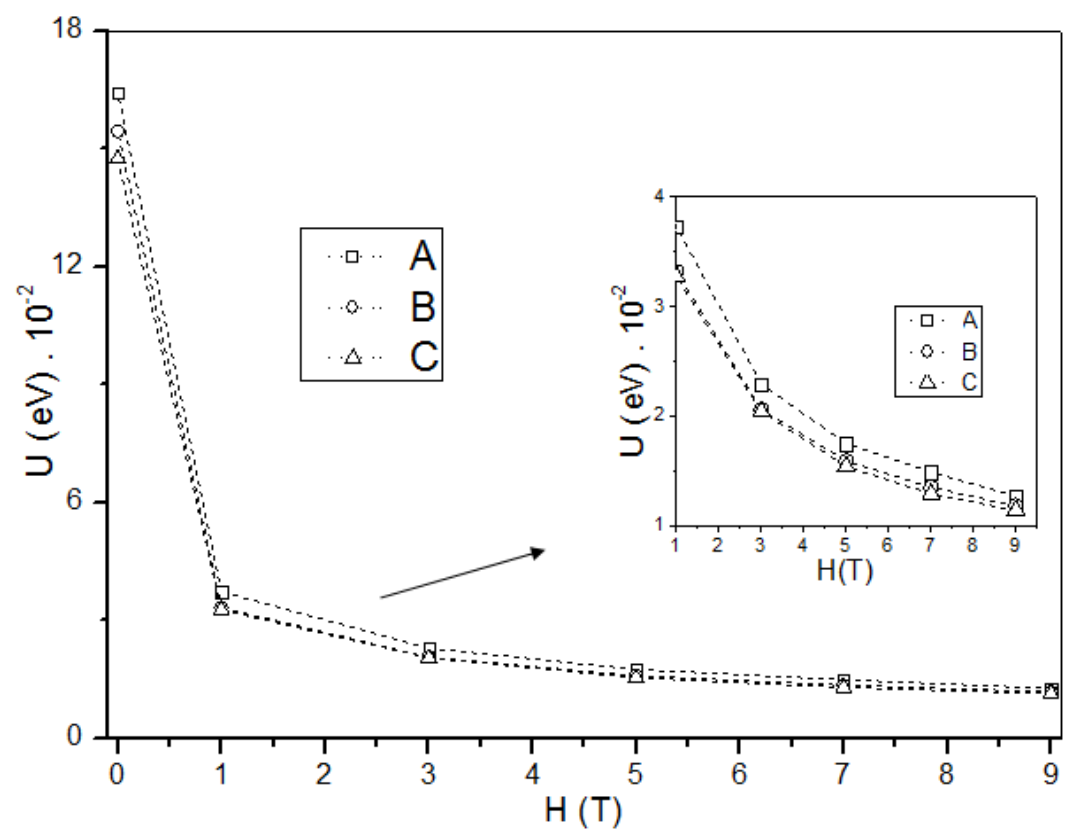


Figure 7

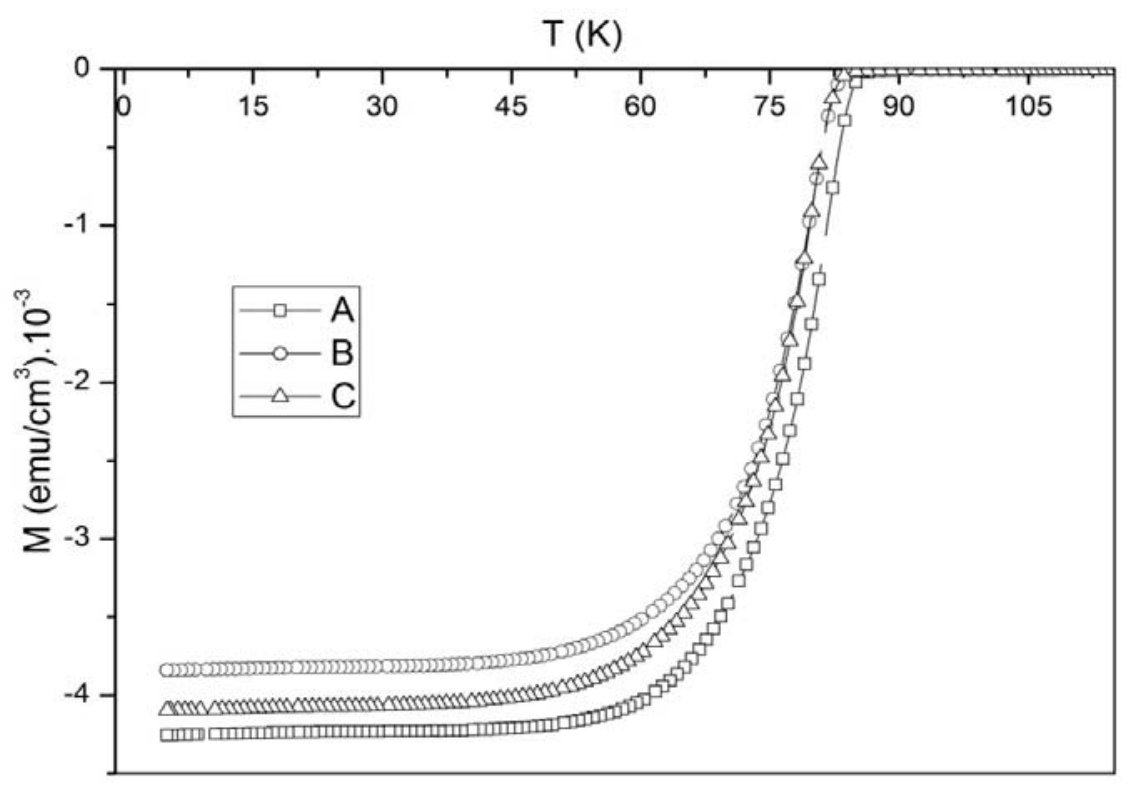


Figure 8

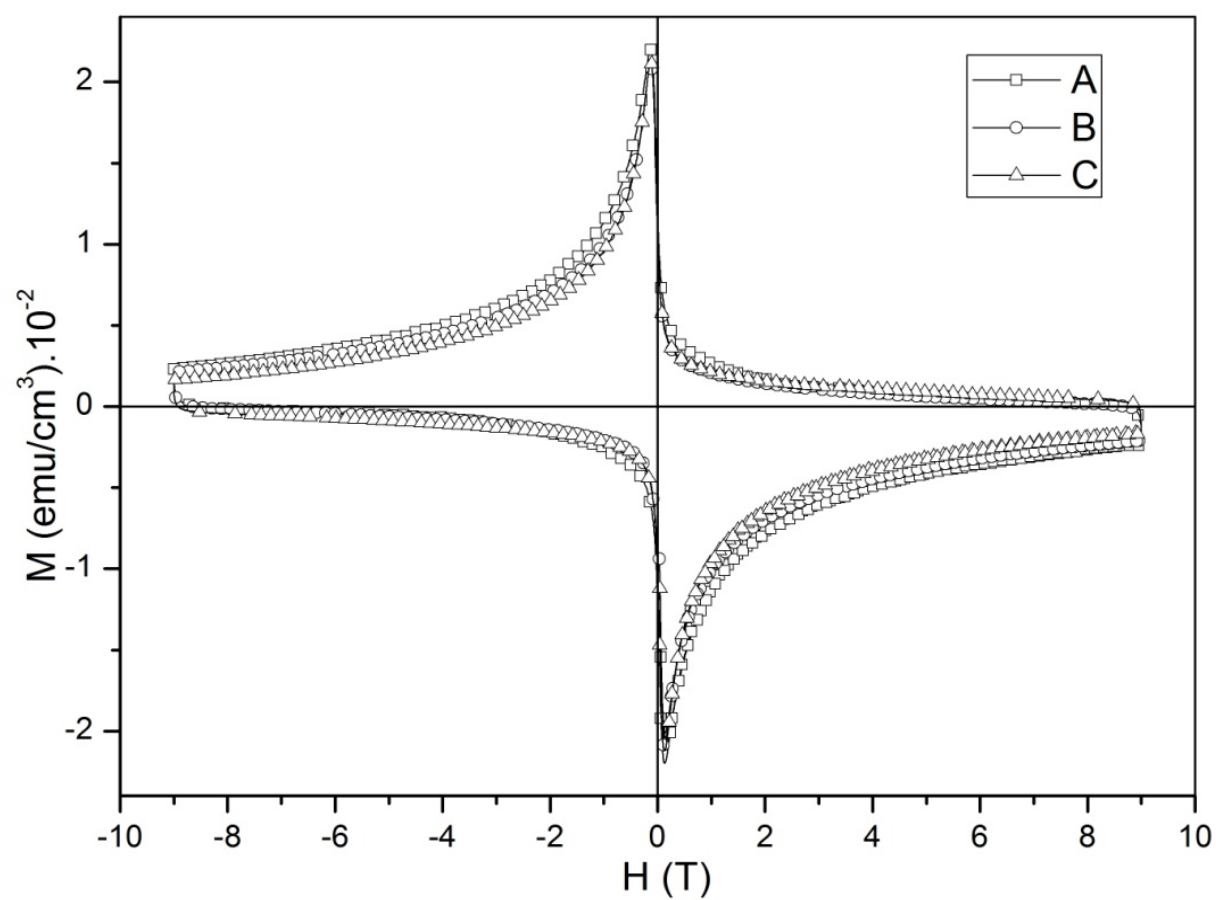


Figure 9

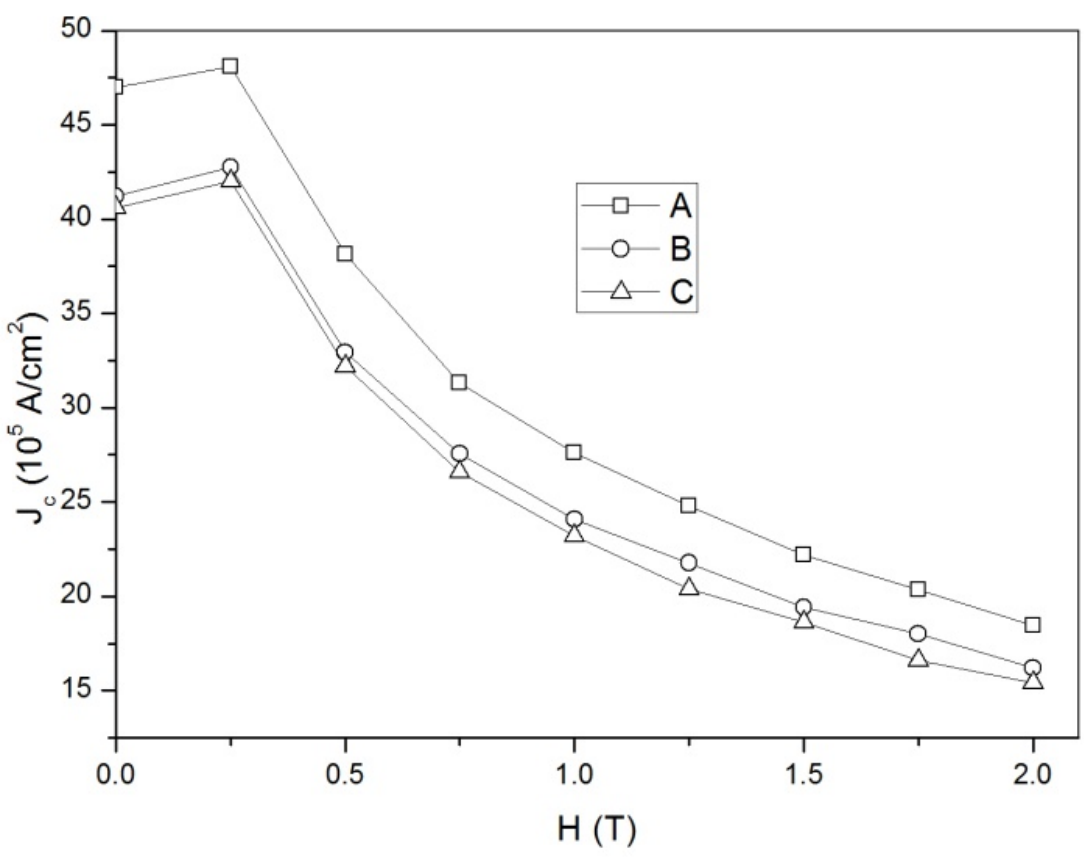


Figure 10

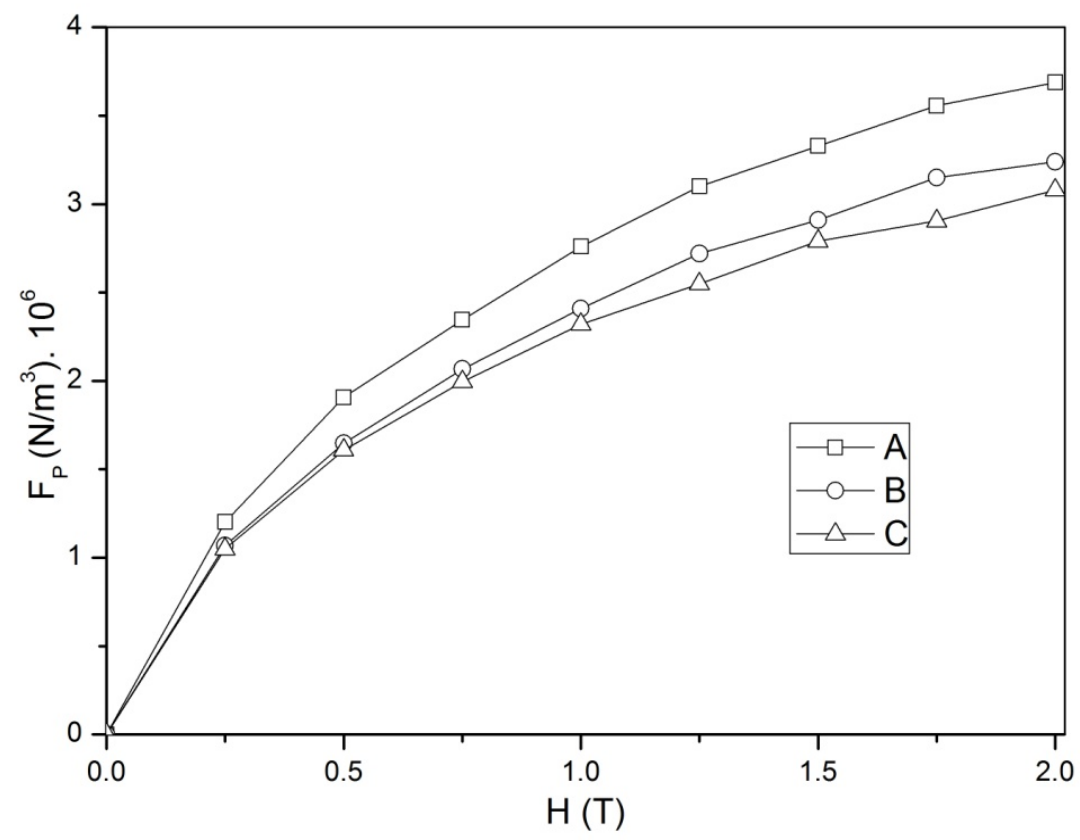


Figure 11

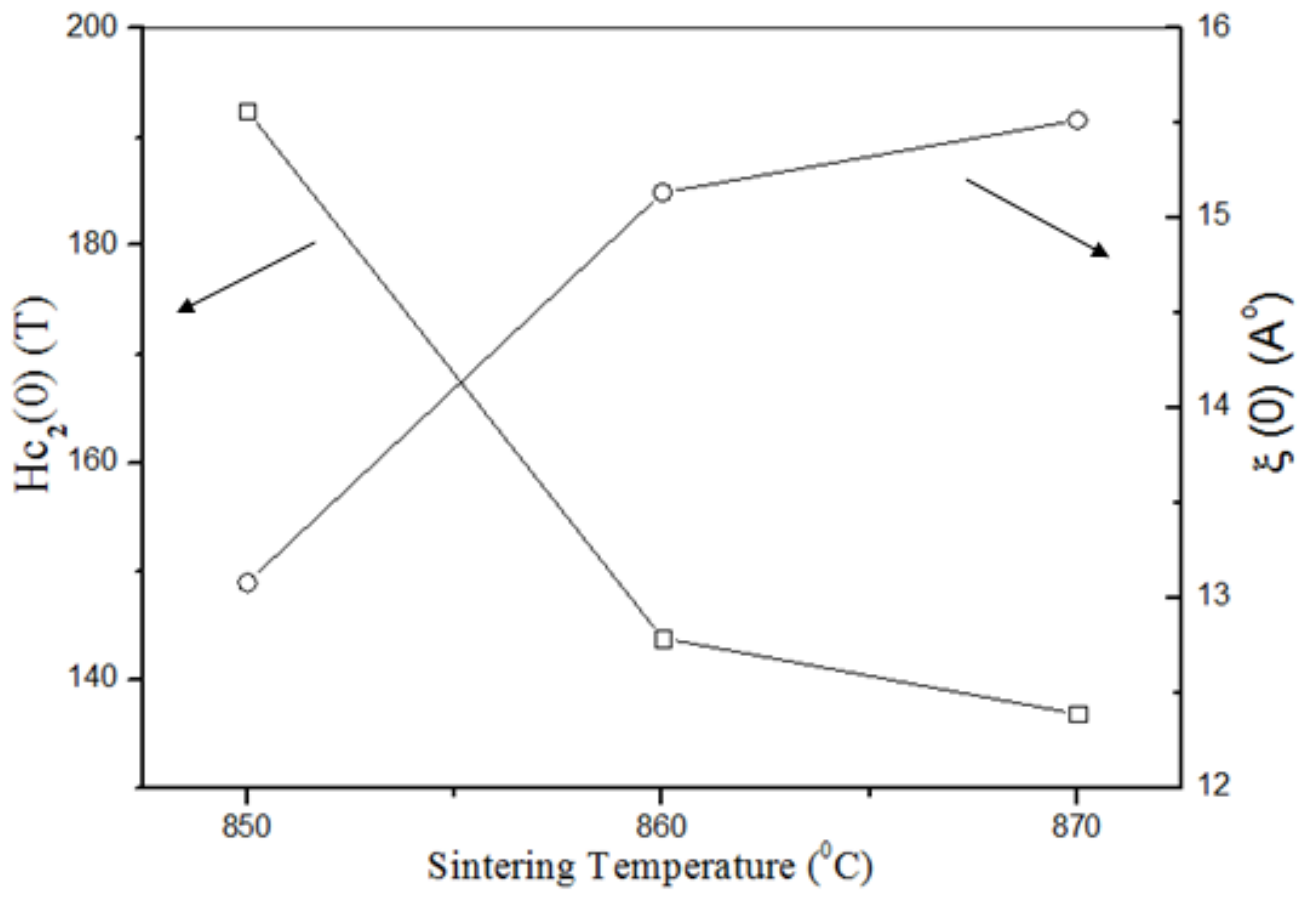

